\title{
METALES PESADOS EN SUELOS DE PLANTACIONES DE CACAO (Theobroma cacao L.) EN TRES REGIONES DEL PERÚ
}

\section{HEAVY METALS IN SOILS OF COCOA PLANTATIONS (Theobroma cacao L.) IN THREE REGIONS OF PERU}

\author{
Enrique Arévalo-Gardini ${ }^{1}$, Meyier E. Obando-Cerpa ${ }^{1}$, Luis B. Zúñiga-Cernades ${ }^{1}$, Cesar O. Arévalo-Hernández ${ }^{1}$, \\ Virupax Baligar ${ }^{2}$ y Zhenli $\mathrm{He}^{3}$
}

\begin{abstract}
Resumen
El cacao ha tenido un crecimiento significativo en los últimos años en el Perú y la presencia de metales pesados en los suelos de estas plantaciones es un potencial problema para las exportaciones de este producto. El objetivo del presente estudio fue determinar los contenidos totales de metales pesados ( $\mathrm{Cd}, \mathrm{Ni}, \mathrm{Pb}, \mathrm{Fe}, \mathrm{Cu}, \mathrm{Zn}, \mathrm{Mn}$ ) en los suelos de plantaciones de cacao en las principales áreas de producción del Perú: Zona Norte (Tumbes, Piura, Cajamarca y Amazonas); Zona central (San Martín, Huánuco y Junín); Zona Sur (Cuzco). Se consideraron plantaciones entre 10 y 15 años de edad. Se realizaron los análisis físicos (textura) y químicos (pH, materia orgánica, CIC, P, K, Ca, Mg, Al, Cd, Ni, Pb, Fe, Cu, Zn, Mn) de los suelos muestreados. Los suelos en el estudio presentan adecuadas condiciones físicas y químicas para el cultivo de cacao. Los valores de metales pesados se encontraron por debajo de lo considerado como fitotóxico. Los valores promedio de hierro, zinc, manganeso, níquel y plomo fueron mayores en la zona sur, mientras que en la zona norte los valores de cobre y cadmio fueron mayores. De forma general el $\mathrm{pH}$, \% de arcilla y $\mathrm{Mg}$ fueron las variables que tuvieron mayor correlación con la concentración de metales pesados.
\end{abstract}

Palabras clave: Cadmio, plomo, suelos contaminados.

\begin{abstract}
Cocoa has experienced significant growth in recent years in Peru and the presence of heavy metals in the soils of these plantations is a potential problem for the export of this product. The objective of the present study was to determine the total concentrations of heavy metals $(\mathrm{Cd}, \mathrm{Ni}$, $\mathrm{Pb}, \mathrm{Fe}, \mathrm{Cu}, \mathrm{Zn}, \mathrm{Mn}$ ) in soils with cacao crops in the main productive areas in Peru: North (Tumbes, Piura, Cajamarca and Amazonas), Center (San Martin, Huánuco and Junín) and South (Cuzco). Contents of heavy metals in soils of cocoa plantations from 10 to 15 years old were evaluated. Physical (texture) and chemical ( $\mathrm{pH}$, organic matter, CEC, P, K, Ca, Mg, Al, Cd, Ni, $\mathrm{Pb}, \mathrm{Fe}, \mathrm{Cu}, \mathrm{Zn}, \mathrm{Mn}$ ) analyzes were conducted in the soils. The results show favorable growing conditions for cocoa. The values found for heavy metals were below those considered as phytotoxic. Mean values of total $\mathrm{Fe}, \mathrm{Zn}, \mathrm{Mn}, \mathrm{Ni}$ and $\mathrm{Pb}$ were higher in the southern region while in the northern region the values of $\mathrm{Cu}$ and $\mathrm{Cd}$ were higher. In general, $\mathrm{pH}$, \% of Clay and $\mathrm{Mg}$ were the variables that had higher significant correlations with heavy metals.
\end{abstract}

Key words: Cadmium, lead, soil, soil contamination.

\section{Introducción.}

El cultivo de cacao (Theobroma cacao L.) ha tenido un crecimiento significativo en la última década, debido a que es una alternativa sostenible para hacer frente a la economía cocalera. En el 2013 se reportaron a nivel nacional 97611 ha sembradas. De éstas los departamentos de Amazonas, Cajamarca, San Martín y Huánuco concentran el 46\% del área sembrada y el 56\% de la producción nacional estimada en 71838 tm (OEEE-MINAG, 2013). Este incremento en las áreas sembradas con cacao, resulta del crecimiento de la demanda por este producto en
América Latina además de mayor calidad e inocuidad de los granos, sobre todo en el contenido de metales pesados importantes en los principales productos derivados del cacao. Los metales pesados están definidos como elementos con un peso específico igual o superior a $5 \mathrm{~g} \mathrm{~cm}-3$ (Khan, 2015). Estos elementos tienen su origen en causas naturales y como consecuencia de actividades antropogénicas tales como los desechos industriales, emisión de gases de los automóviles y las practicas agronómicas (Aikpokpodion, 2012). Sin embargo, para que estos elementos sean absorbidos por las plantas existen 
varios factores, entre los que se cuentan, por ejemplo, los atributos del suelo que juegan un papel importante en reducir o aumentar la toxicidad de los metales en el suelo (Puga et al., 2006). Dentro de los principales atributos que están relacionados con la movilidad y disponibilidad de metales pesados en el suelo se encuentran el $\mathrm{pH}$, la materia orgánica, los óxidos de Fe y Mn y el contenido de arcilla (Alloway, 2013). Además la alta concentración de nutrientes puede afectar la disponibilidad de algunos elementos como ocurre en la interacción entre $\mathrm{P}$ y As, donde el As disminuye con un mayor valor de P (Violante \& Pigna, 2002).

Recientes estudios han indicado que los suelos y granos en áreas de cultivo de cacao podrían presentar altos niveles de metales pesados (Crozier et al., 2012, Huamaní et al., 2012) lo que podría llevar a problemas de consumo y exportación de este producto en el futuro. Los metales pesados del suelo una vez disponibles, pueden ser absorbidos por la planta de cacao; sin embargo, su distribución en la planta y su acumulación es variable. En el caso del cadmio, en la actualidad, no se conoce un papel fisiológico definido en la planta pero puede concentrarse en las raíces, brotes, hojas o partes comestibles como los granos por su constitución grasosa (Augstburger et al., 2000, Rascio \& Navari-Izzo, 2011).

Debido a los riesgos para la salud humana que implican altas concentraciones de metales pesados en el suelo y las plantas, como es el caso del cacao, considerado un cultivo bandera en el Perú, el presente trabajo se realizó con el objetivo de determinar los contenidos de metales pesados en los suelos de plantaciones de cacao en las principales áreas de producción del Perú: Zona Norte (Tumbes, Piura, Cajamarca y Amazonas); Zona Central (San Martín, Huánuco y Junín) y Zona Sur (Cuzco).

\section{Materiales y métodos.}

El estudio se realizó en 70 plantaciones de cacao entre 10 a 15 años de edad localizadas en la Zona Norte (Regiones de Tumbes, Piura, Cajamarca, Amazonas); Zona Centro (Regiones de Huánuco, San Martín y Junín) y Zona Sur (Región Cuzco) - Perú (Figura1).

\section{Muestreo de suelos}

Las muestras de suelo usadas para este estudio fueron colectadas a seis profundidades $(0-5 \mathrm{~cm}, 5-10$ $\mathrm{cm}, 10-20 \mathrm{~cm}, 20-40 \mathrm{~cm}, 40-60 \mathrm{~cm}, 60-80 \mathrm{~cm}$ ) entre febrero y abril del 2014. Cada muestra estuvo constituida por un kilogramo de mezcla de suelos en cada profundidad, tomado de ocho calicatas de hasta $80 \mathrm{~cm}$ de profundidad distribuidas al azar en forma de zigzag dentro de cada plantación. La hojarasca superficial de cada punto de muestreo fue removida previamente a la colecta de las muestras. Las muestras fueron transportadas al laboratorio donde fueron secadas al aire, molidas y pasadas por un tamiz de 2 mm de diámetro y almacenadas a temperatura ambiente.

Determinación de las propiedades físicas y químicas del suelo

El análisis de las propiedades físicas y químicas fue realizado en el laboratorio analítico de plantas y suelos del Instituto de Cultivos Tropicales-ICT, Tarapoto, siguiendo los protocolos recomendados por Anderson \& Ingram (1993). La textura del suelo fue determinada con el densímetro de Bouyoucos usando $\mathrm{NaOH} 1 \mathrm{~mol} \mathrm{~L}^{-1}$ como dispersante. Las siguientes propiedades químicas fueron determinadas: pH (1: 2 $\mathrm{H}_{2} \mathrm{O}$; método del potenciómetro), conductividad eléctrica (conductímetro), iones disponibles ( $\mathrm{P}, \mathrm{K}$; método de Olsen modificado), iones totales $(\mathrm{Cd}, \mathrm{Ni}$, $\mathrm{Pb}, \mathrm{Fe}, \mathrm{Cu}, \mathrm{Zn}, \mathrm{Mn}$ ) por el método de digestión acida EPA-3050B (USEPA, 1996), bases intercambiables $(\mathrm{K}, \mathrm{Ca}, \mathrm{Mg}$ ) para suelos con $\mathrm{pH} \leq 5.5$ por $1 \mathrm{M}$ acetato de amonio y para suelos con $\mathrm{pH}>5.5$ por $1 \mathrm{~N} \mathrm{KCl}$ (Anderson \& Ingram, 1993), acidez intercambiable $(\mathrm{Al}+\mathrm{H})$ por el método de Yuan (Yuan, 1959), materia orgánica del suelo (MOS) por el método de Walkley y Black. Ca, Mg y $\mathrm{K}$ en los extractantes fueron determinados por espectrofotómetro de absorción atómica, $\mathrm{P}$ en el extractante por colorimetría con Molibdato de amonio-ácido ascórbico (Anderson \& Ingram, 1993). La capacidad de intercambio catiónico (CIC) fue calculada por la suma de bases intercambiables y la acidez intercambiable $(\mathrm{Al}+\mathrm{H})$ (Anderson \& Ingram, 1993). Para asegurar la precisión y calidad de los resultados se contrastaron con reactivo blanco y material de referencia certificado SS-2 para suelos con bajo nivel de contaminantes y AG-1 para suelos arcillosos, obtenido de SCP Science (EnviroMAT ${ }^{\mathrm{TM}} \&$ AgroMAT $^{\mathrm{TM}}$ ), que fueron preparados $\mathrm{y}$ analizados con el mismo procedimiento descrito anteriormente.

\section{Análisis Estadístico}

Todos los análisis estadísticos fueron realizados usando InfoStat, version 2014 (Di Rienzo, 2014). Las propiedades físico químicas de los suelos por departamento, provincias y profundidades de muestreo fueron comparadas por medio del análisis de variancia (ANVA) y las medias fueron comparadas por la prueba de Scott \& Knott $(p<0.05)$. También se realizaron análisis de correlación de Pearson entre las propiedades evaluadas y el contenido de metales pesados. Los resultados de la caracterización físico química de los suelos así como el contenido de metales pesados y sus respectivas correlaciones, fueron analizados hasta la profundidad de $20 \mathrm{~cm}$.

\section{Resultados.}

Atributos de suelo en las plantaciones de cacao

Los resultados de los atributos de suelo evaluados en plantaciones de cacao en las regiones estudiadas a la profundidad de 0 a $20 \mathrm{~cm}$, son presentados en la Tabla 1. Se encontró una amplia diversidad textural en 
cada una de las zonas estudiadas: desde suelos francos hasta arcillosos. Se observaron diferencias estadísticamente significativas $(\mathrm{p}<0.05)$ para el $\mathrm{pH}$, MOS, $\mathrm{P}$ disponible; CIC y los iones intercambiables $\mathrm{Ca}^{2+}, \mathrm{Mg}^{2+}$. El $\mathrm{pH}$ de los suelos fluctuó entre fuertemente ácidos ( $\mathrm{pH}=5.38)$ a moderadamente alcalinos $(\mathrm{pH}=8.29)$. Los más altos $(\mathrm{pH}>7)$ fueron encontrados en plantaciones de cacao de las regiones Tumbes, Piura (Morropón y Piura), Cajamarca y San Martín (Bellavista, Huallaga y Mariscal Cáceres); los valores menores $(\mathrm{pH}<7)$ en Piura (Huancabamba), Amazonas, San Martín (El Dorado y Tocache), Huánuco, Junín y Cuzco. Los valores alcalinos de los suelos evaluados posiblemente se deban a la mayor concentración de calcio en estos suelos. La Unión Europea propuso las directivas de Kelley (Acevedo, 2005) donde el pH típico en suelos ácidos no contaminados con metales pesados fluctúa entre 6 y 7; ligera contaminación se da entre $\mathrm{pH}$ de 5-6 y conforme el pH disminuye la contaminación se incrementa. En suelos alcalinos no contaminados por metales pesados el $\mathrm{pH}$ se encuentra entre $>7$ a 8 , ligeras contaminaciones se dan entre $\mathrm{pH} 8$ y 9 y la contaminación en este tipo de suelos se incrementa en la medida que se incrementa el $\mathrm{pH}$. Valores medios de MOS (2 - 4\%) fueron encontrados en Tumbes (Tumbes), Piura, Cajamarca (Jaén), Amazonas y San Martín (Bellavista, El Dorado, Huallaga y Mariscal Cáceres), Huánuco y Junín; concentraciones altas (>4\%) se hallaron en la Región Cuzco; mientras que bajos contenidos se localizaron en Tumbes (Zarumilla) y San Martín (Tocache). La materia orgánica, de forma general, influencia en las propiedades del suelo que contribuyen a la calidad de los suelos como la densidad aparente, estructura, acidez, disponibilidad de nutrientes y disminución de la disponibilidad de metales pesados (Bot \& Benitez 2005; Alloway, 2013).

Los valores medios de $\mathrm{P}$ disponible $\left(>14 \mu \mathrm{g} \mathrm{g}^{-1}\right.$ ) se encontraron en Tumbes (Zarumilla), Piura (Piura y Morropón), Cajamarca (San Ignacio) y Cuzco; los valores medios se registraron en Tumbes (Tumbes), Cajamarca (Jaen), San Martín (Bellavista y Mariscal Cáceres), Huánuco (Huamalíes) y Junín; en las demás plantaciones los valores de $\mathrm{P}$ fueron bajos $\left(<7 \mu \mathrm{g} \mathrm{g}^{-1}\right)$; en general la mayoría de los suelos evaluados presentaron disponibilidad de $\mathrm{P}$ por debajo del nivel crítico de $10 \mu \mathrm{g} \mathrm{g}^{-1}$ para suelos de cacao propuesto por Aikpokpodion (2010). De 6 a 8\% del fosforo disponible en el suelo es removido por los granos de cacao, por lo que estos suelos necesitan ser fertilizados para suplir estas deficiencias (Ogunlade \& Aikpokpodion, 2006).

Respecto al $\mathrm{K}$ disponible, se encontraron valores medios (100-200 $\mathrm{\mu g} \mathrm{g}^{-1}$ ) en Tumbes (Tumbes), Piura (Piura), Cajamarca (San Ignacio), Amazonas (Bagua), San Martín (Bellavista y Mariscal Cáceres) y en Junín. Los demás sitios evaluados presentaron niveles de $\mathrm{K}$ disponible bajos $\left(<100 \mu \mathrm{g} \mathrm{g}^{-1}\right)$. El contenido de potasio intercambiable en los suelos estudiados fluctuó entre $0.13 \pm 0.03$ y $0.62 \pm 0.03 \mathrm{cmol} \mathrm{kg}^{-1}$. Todos los suelos mostraron valores mayores al nivel crítico de potasio intercambiable $\left(0.03 \mathrm{cmol} \mathrm{kg}^{-1}\right)$ para plantaciones de cacao, lo que es adecuado para los suelos estudiados (Aikpokpodion, 2010). El potasio es el macronutriente más requerido por la planta de cacao; sin embargo, es un ion bastante móvil, que se pierde fácilmente a través de la escorrentía y la lixiviación de los suelos (Giron et al., 2001; Aikpokdion, 2010). La CIC es un buen indicador de la fertilidad de un suelo al estar relacionado a una mayor adsorción de iones y menor pérdida de nutrientes por lixiviación. En nuestro estudio los valores fluctuaron entre $4.75 \pm 1.12$ y $41.09 \pm 1.12 \mathrm{cmol} \mathrm{kg}^{-1}$ para los suelos de San Martín (El Dorado y Bellavista respectivamente). Los mayores valores de CIC están relacionados posiblemente al mayor contenido de arcilla y materia orgánica en estos suelos (Ololade et al. 2010).

Los valores de calcio fluctuaron entre $2.99 \pm 0.23$ y $38.69 \pm 1.35 \mathrm{cmol} \mathrm{kg}^{-1}$ en San Martín (Tocache y Bellavista respectivamente). Los valores altos de calcio están relacionados a los valores altos de $\mathrm{pH}$ en similares zonas de producción de cacao.

El contenido de magnesio intercambiable en los suelos estudiados varió entre $0.35 \pm 0.16$ y $6.71 \pm 0.08$ cmol kg ${ }^{-1}$. Los suelos de San Martín (El Dorado, Tocache) y Huánuco (Huamalíes) mostraron valores de magnesio intercambiable por debajo de $0.8 \mathrm{cmol} \mathrm{kg}$ -1 que es el nivel crítico para suelos con cacao (Aikpokpodion, 2010). La acidez intercambiable $\left(\mathrm{Al}^{3+}+\mathrm{H}^{+}\right)$varió entre 0.00 y $0.74 \pm 0.62 \mathrm{cmol} \mathrm{kg}^{-1}$. Los valores altos de $\left(\mathrm{Al}^{3+}+\mathrm{H}^{+}\right)$coinciden con los valores bajos de $\mathrm{pH}$, siendo el aluminio, debido a su hidrólisis, la causa indirecta de acidez de los suelos en Amazonas, San Martín, Huánuco y Cuzco. Estas condiciones fueron encontradas en estudios anteriores realizados por Huamaní et al. (2012) en suelos cacaoteros del Huallaga en el departamento de Huánuco.

Contenido de metales pesados en suelos cacaoteros

En la Tabla 2 se muestran los valores de metales pesados totales (Fe, $\mathrm{Cu}, \mathrm{Zn}, \mathrm{Mn}, \mathrm{Cd}, \mathrm{Ni}, \mathrm{Pb}$ ), analizados por departamento y provincias a profundidad de 0 a $20 \mathrm{~cm}$. Se encontraron diferencias estadísticas significativas $(\mathrm{p}<0.05)$ para todos los elementos en estudio $\mathrm{y}$ en todas las zonas muestreadas.

Los valores de hierro fluctuaron entre $0.21 \pm 0.07 \mathrm{x}$ $10^{4} \mu \mathrm{g} \mathrm{g}^{-1}$ en San Martín (El Dorado) y 4.28 $\pm 0.01 \mathrm{x}$ $10^{4} \mathrm{\mu g} \mathrm{g}^{-1}$ en el Cuzco; valores intermedios se registraron en las otras provincias de las diferentes regiones estudiadas. Los valores de hierro están entre los rangos sugeridos para suelos agrícolas (Alloway, 2013). En promedio los valores fueron mayores en la Región Sur (4.28 x $\left.10^{4} \mu g^{-1}\right)$, seguido de la Región 
Tabla 2. Valores de metales pesados totales en suelos de plantaciones de cacao por Departamentos y Provincias estudiadas a profundidad de $0-20 \mathrm{~cm}$.

\begin{tabular}{|c|c|c|c|c|c|c|c|}
\hline Departamento & $\mathbf{F e}^{*}$ & $\mathbf{C u}$ & Zn & Mn & Cd & $\mathbf{N i}$ & $\mathbf{P b}$ \\
\hline \multicolumn{8}{|l|}{ Tumbes } \\
\hline$\overline{\text { Tumbes }}$ & $3.31 \pm 0.03 a^{* *}$ & $43.16 \pm 0.98 a$ & $111.72 \pm 0.92 \mathrm{a}$ & $419.84 \pm 9.99 c$ & $0.50 \pm 0.03 a$ & $27.67 \pm 1.08 \mathrm{a}$ & $15.84 \pm 0.20 \mathrm{a}$ \\
\hline $\begin{array}{l}\text { Zarumilla } \\
\text { Piura }\end{array}$ & $1.55 \pm 0.03 b$ & $16.48 \pm 0.98 b$ & $45.83 \pm 0.92 b$ & $379.86 \pm 9.99 c$ & $0.05 \pm 0.03 c$ & $13.54 \pm 1.08 b$ & $9.67 \pm 0.20 b$ \\
\hline$\overline{\text { Huamcabamba }}$ & $3.04 \pm 0.02 \mathrm{a}$ & $49.42 \pm 0.66 a$ & $72.42 \pm 0.61 \mathrm{a}$ & $725.76 \pm 6.66 b$ & $0.14 \pm 0.02 b$ & $42.12 \pm 0.72 \mathrm{a}$ & $7.95 \pm 0.14 b$ \\
\hline Morropón & $2.11 \pm 0.02 \mathrm{a}$ & $27.46 \pm 0.66 b$ & $64.80 \pm 0.61 a$ & $429.73 \pm 6.66 c$ & $0.53 \pm 0.02 a$ & $10.05 \pm 0.72 b$ & $9.18 \pm 0.14 b$ \\
\hline $\begin{array}{l}\text { Piura } \\
\text { Cajamarca } \\
\end{array}$ & $3.32 \pm 0.01 \mathrm{a}$ & $49.14 \pm 0.28 a$ & $85.93 \pm 0.26 a$ & $671.85 \pm 2.86 b$ & $0.48 \pm 0.01 a$ & $25.30 \pm 0.30 a$ & $12.67 \pm 0.06 a$ \\
\hline Jaen & $2.9 \pm 0.02 \mathrm{a}$ & $53.58 \pm 0.66 a$ & $70.56 \pm 0.61 a$ & $795.8 \pm 6.66 b$ & $0.01 \pm 0.02 c$ & $6.25 \pm 0.72 b$ & $11.09 \pm 0.14 b$ \\
\hline $\begin{array}{l}\text { San Ignacio } \\
\text { Amazonas }\end{array}$ & $2.21 \pm 0.03 a$ & $29.59 \pm 0.98 b$ & $61.00 \pm 0.92 \mathrm{a}$ & $553.19 \pm 9.99 c$ & $0.00 \pm 0.00 c$ & $8.29 \pm 1.08 b$ & $8.47 \pm 0.20 b$ \\
\hline$\overline{\text { Bagua }}$ & $2.50 \pm 0.00 \mathrm{a}$ & $25.5 \pm 0.13 b$ & $75.34 \pm 0.12 a$ & $524.87 \pm 1.32 c$ & $0.11 \pm 0.00 \mathrm{~b}$ & $16.56 \pm 0.14 a$ & $10.05 \pm 0.03 b$ \\
\hline $\begin{array}{l}\text { Condorcanqui } \\
\text { San Martín }\end{array}$ & $3.06 \pm 0.01 a$ & $43.56 \pm 0.32 \mathrm{a}$ & $81.54 \pm 0.30 a$ & $739.84 \pm 3.31 b$ & $0.01 \pm 0.01 c$ & $22.75 \pm 0.36 a$ & $15.92 \pm 0.07 a$ \\
\hline Bellavista & $1.66 \pm 0.07 b$ & $15.44 \pm 1.96 b$ & $52.85 \pm 1.85 b$ & $486.64 \pm 19.89 c$ & $0.20 \pm 0.06 b$ & $13.69 \pm 2.13 b$ & $7.18 \pm 0.41 b$ \\
\hline El Dorado & $0.21 \pm 0.07 b$ & $2.43 \pm 1.96 b$ & $3.76 \pm 1.85 b$ & $82.63 \pm 19.89 c$ & $0.00 \pm 0.00 c$ & $1.64 \pm 2.13 b$ & $5.52 \pm 0.41 b$ \\
\hline $\begin{array}{l}\text { Huallaga } \\
\text { Mariscal }\end{array}$ & $1.56 \pm 0.07 b$ & $14.44 \pm 1.96 b$ & $43.56 \pm 1.85 b$ & $482.68 \pm 19.89 c$ & $0.13 \pm 0.06 b$ & $10.89 \pm 2.13 b$ & $5.76 \pm 0.41 b$ \\
\hline Cáceres & $1.58 \pm 0.01 b$ & $14.44 \pm 0.4 b$ & $49.00 \pm 0.37 b$ & $517.56 \pm 4.00 c$ & $0.21 \pm 0.01 b$ & $11.63 \pm 0.44 b$ & $9.36 \pm 0.08 b$ \\
\hline $\begin{array}{l}\text { Tocache } \\
\text { Huánuco }\end{array}$ & $1.26 \pm 0.01 b$ & $6.86 \pm 0.32 b$ & $35.16 \pm 0.30 b$ & $477.42 \pm 3.31 c$ & $0.00 \pm 0.00 c$ & $3.50 \pm 0.36 b$ & $6.71 \pm 0.07 b$ \\
\hline$\overline{\text { Huamalíes }}$ & $4.11 \pm 0.07 a$ & $49 \pm 1.96 a$ & $87.24 \pm 1.85 a$ & $919.3 \pm 19.89 b$ & $0.00 \pm 0.00 c$ & $43.03 \pm 2.13 a$ & $15.37 \pm 0.41 a$ \\
\hline $\begin{array}{l}\text { Leoncio Prado } \\
\text { Junin }\end{array}$ & $2.34 \pm 0.02 \mathrm{a}$ & $21.34 \pm 0.66 b$ & $55.06 \pm 0.61 b$ & $876.16 \pm 6.66 b$ & $0.00 \pm 0.00 c$ & $17.22 \pm 0.72 \mathrm{a}$ & $6.50 \pm 0.14 b$ \\
\hline $\begin{array}{l}\overline{\text { Satipo }} \\
\text { Cuzco } \\
\end{array}$ & $2.32 \pm 0.02 \mathrm{a}$ & $21.53 \pm 0.49 b$ & $73.10 \pm 0.46 a$ & $615.04 \pm 4.97 c$ & $0.10 \pm 0.01 b$ & $19.10 \pm 0.53 a$ & $12.89 \pm 0.10 \mathrm{a}$ \\
\hline La Convención & $4.28 \pm 0.01 \mathrm{a}$ & $34.46 \pm 0.4 a$ & $96.83 \pm 0.37 a$ & $1275.20 \pm 4.00 \mathrm{a}$ & $0.00 \pm 0.00 c$ & $24.70 \pm 0.44 a$ & $21.81 \pm 0.08 a$ \\
\hline$P v$ & $<0.0001$ & 0.0035 & $<0.0001$ & 0.0001 & 0.0001 & 0.005 & 0.0005 \\
\hline
\end{tabular}

Norte $\left(2.67 \times 10^{4} \mu \mathrm{g} \mathrm{g}^{-1}\right)$, tendencias que coinciden con los resultados de Crozier et al. (2012) en un estudio de metales pesados realizado en Perú y Venezuela en suelo y granos de cacao, reportando valores promedios de Fe de $3.4 \times 10^{4} \mathrm{\mu g} \mathrm{g}^{-1}$ para Amazonas, Cajamarca Tumbes y Piura y de $3.2 \times 10^{4}$ $\mu \mathrm{g} \mathrm{g}^{-1}$ para San Martín y Huánuco.

El cobre se encontró entre $2.43 \pm 1.96 \mu \mathrm{g} \mathrm{g}^{-1}$ en San Martín (El Dorado) y 53.58 $\pm 0.66 \mu \mathrm{g} \mathrm{g} \mathrm{g}^{-1}$ en Cajamarca (Jaén). En términos promedios, en la Zona Norte y Sur, los valores de Cu fueron mayores (37.54 y $34.46 \mu \mathrm{g} \mathrm{g}^{-1}$ respectivamente) a la Zona Central (17.7 $\left.\mu \mathrm{g} \mathrm{g}^{-1}\right)$. Estos resultados concuerdan con lo reportado por Crozier et al. (2012) para este metal en el suelo. Según las directivas de Kelley (Acevedo et al., 2005) los valores típicos de cobre para suelos no contaminados fluctúa entre 0 y $100 \mu \mathrm{g} \mathrm{g}^{-1}$.

El zinc en los suelos evaluados en San Martín (El Dorado) fue menor $\left(3.76 \pm 1.85 \mu \mathrm{g} \mathrm{g}^{-1}\right)$ y en Tumbes (Tumbes) fue mayor $\left(111.72 \pm 0.92 \mu \mathrm{g} \mathrm{g}^{-1}\right)$; el promedio del Zn en la Zona Norte $\left(74.35 \mu \mathrm{g} \mathrm{g}^{-1}\right)$ fue mayor a la Zona Central (49.97 $\mu \mathrm{g} \mathrm{g}^{-1}$ ) y Zona Sur (96.83 $\mu \mathrm{g} \mathrm{g}^{-1}$ ). Crozier et al. (2012) reportaron valores mayores en la zona norte del Perú, coincidentes con lo encontrado en nuestro estudio. En todos los casos los valores obtenidos están por debajo de los niveles excesivamente fitotóxicos de 200 a 400 $\mu g^{-1}$ (Cameron, 1992) y también según las directivas de Kelly (Acevedo et al., 2005) los valores típicos para suelos no contaminados con zinc están entre 0 y $250 \mu \mathrm{g} \mathrm{g}^{-1}$.

Los valores de manganeso fluctuaron entre 82.63 $\pm 19.89 \mu \mathrm{g} \mathrm{g}^{-1}$ y $1275.20 \pm 4.00 \mu \mathrm{g} \mathrm{g}^{-1}$ en San Martín (El Dorado) y Cuzco respectivamente. Cameron (1992) indica que los valores máximos permisibles para manganeso están entre 3000 a 8000 $\mu \mathrm{g} \mathrm{g}^{-1}$, lo que varía respecto a las directivas de Kelly (Acevedo et al., 2005) donde los valores típicos de manganeso en suelos no contaminados fluctúa entre 0 y $500 \mu \mathrm{g} \mathrm{g}^{-1}$. En este grupo se hallan los suelos de Tumbes, Piura (Morropón), San Martín (Bellavista, El Dorado, Huallaga y Tocache). Suelos de ligera contaminación con valores de manganeso entre 500 y $1000 \mu \mathrm{g} \mathrm{g}^{-1}$ se encuentran en Piura (Huancabamba y Piura), Cajamarca, Amazonas, San Martin (Mariscal Cáceres), Huánuco y Junín; y suelos contaminados de manganeso con valores entre 1000 y $2000 \mu \mathrm{g} \mathrm{g}^{-1}$ se encuentran en Cuzco (La Convención). 
Tabla 3. Valores de metales pesados totales a diferentes profundidades del suelo en plantaciones de cacao de las regiones estudiadas.

\begin{tabular}{|c|c|c|c|c|c|c|c|}
\hline Profundidad & $\mathbf{F e}^{*}$ & $\mathrm{Cu}$ & Zn & Mn & Cd & $\mathbf{N i}$ & $\mathbf{P b}$ \\
\hline $0-5$ & $2.47 \pm 0.00$ & $25.40 \pm 0.04$ & $72.59 \pm 0.04$ & $654.85 \pm 0.45 a^{* *}$ & $0.10 \pm 0.00 \mathrm{a}$ & $16.56 \pm 0.04$ & $11.70 \pm 0.01$ \\
\hline $5-10$ & $2.47 \pm 0.00$ & $27.88 \pm 0.06$ & $67.40 \pm 0.04$ & $620.01 \pm 0.53 a$ & $0.07 \pm 0.00 \mathrm{a}$ & $16.32 \pm 0.04$ & $10.96 \pm 0.01$ \\
\hline $10-20$ & $2.52 \pm 0.00$ & $27.88 \pm 0.06$ & $66.26 \pm 0.04$ & $599.76 \pm 0.53 a$ & $0.05 \pm 0.00 \mathrm{a}$ & $16.65 \pm 0.04$ & $10.89 \pm 0.01$ \\
\hline $20-40$ & $2.61 \pm 0.00$ & $28.73 \pm 0.06$ & $64.16 \pm 0.04$ & $551.31 \pm 0.53 b$ & $0.03 \pm 0.00 b$ & $17.81 \pm 0.04$ & $10.43 \pm 0.01$ \\
\hline $40-60$ & $2.61 \pm 0.00$ & $29.38 \pm 0.06$ & $63.52 \pm 0.05$ & $503.10 \pm 0.56 b$ & $0.03 \pm 0.00 \mathrm{~b}$ & $17.72 \pm 0.05$ & $10.24 \pm 0.01$ \\
\hline $60-80$ & $2.66 \pm 0.00$ & $29.16 \pm 0.06$ & $63.04 \pm 0.05$ & $501.31 \pm 0.58 b$ & $0.02 \pm 0.00 b$ & $18.15 \pm 0.05$ & $10.05 \pm 0.01$ \\
\hline & 0.8833 & 0.8560 & 0.3384 & 0.0052 & 0.0038 & 0.9677 & 0.5024 \\
\hline
\end{tabular}

*, Los valores de Fe están expresados en $10^{4}$ miles de $\mu \mathrm{g} \mathrm{g}^{-1}$.

**, Promedios unidos con igual letra en columna no difieren significativamente, según Scott \& Knott Alfa=0.05

El cadmio ha sido el metal menos frecuente en los suelos muestreados. Valores nulos de $0.00 \mu \mathrm{g} \mathrm{g}^{-1}$ se encontraron en Cajamarca (San Ignacio), San Martín (El Dorado y Tocache), Huánuco y Cuzco y el mayor valor de $0.53 \pm 0.02 \mu \mathrm{g} \mathrm{g}^{-1}$ se encontró en Piura (Morropón). En promedio los valores de cadmio se encontraron mayores en la Zona Norte $\left(0.20 \mu \mathrm{g} \mathrm{g}^{-1}\right)$, seguido de la Zona Central $\left(0.08 \mu \mathrm{g} \mathrm{g}^{-1}\right)$ y Zona Sur $\left(0.00 \mu \mathrm{g} \mathrm{g}^{-1}\right)$. Estos resultados podrían deberse a la amplitud de las áreas cacaoteras y que en la aleatorización se han tomado plantaciones con bajo o nulo contenido de cadmio; sin embargo, la tendencia de la presencia de $\mathrm{Cd}$ en el suelo de las zonas en estudio coincide con Crozier et al. (2012), quienes encontraron valores más altos de Cd en la Zona Norte $\left(0.79 \mu \mathrm{g} \mathrm{g}^{-1}\right)$ y menores en la Zona Central $(0.68 \mu \mathrm{g} \mathrm{g}$ $\left.{ }^{-1}\right)$ y Sur (0.46 $\left.\mu \mathrm{g} \mathrm{g}^{-1}\right)$, Huamaní et al. (2012) encontró en suelos de Huánuco y Ucayali, que corresponde a la Zona Centro de nuestro estudio, valores de cadmio promedio de $0.53 \mu \mathrm{g} \mathrm{g}^{-1} \mathrm{y}$ considera a estos de bajo contenido. La Agencia del Medio Ambiente de los Estados Unidos de Norteamérica (USEPA) estableció como nivel crítico en $0.43 \mu \mathrm{g} \mathrm{g}^{-1}$ de cadmio total en suelos agrícolas (USEPA, 2002). En base a ello los valores obtenidos para $\mathrm{Cd}$ en la mayoría de suelos muestreados, con la excepción de los valores de Tumbes [Tumbes $\left(0.50 \pm 0.03 \mu \mathrm{g} \mathrm{g}^{-1}\right)$ ] y Piura [Morropón $\left(0.53 \pm 0.02 \mu \mathrm{g} \mathrm{g}^{-1}\right)$ y Piura $(0.48 \pm 0.01 \mu \mathrm{g} \mathrm{g}$ $\left.{ }^{-1}\right)$ ] están por debajo de los valores sugeridos como críticos para la USEPA o como excesivamente fitotóxicos (3 a $10 \mu \mathrm{g} \mathrm{g}^{-1}$ ) sugerido por Cameron

Tabla 4. Correlación de Pearson entre los metales pesados totales y las principales características de los suelos de plantaciones de cacao estudiados.

\begin{tabular}{|c|c|c|c|c|c|c|c|c|c|c|c|}
\hline $\begin{array}{l}\text { Metal } \\
\text { Pesado }\end{array}$ & nid. & $\begin{array}{c}\text { pH } \\
(1: 2 \\
\left.\mathrm{H}_{2} \mathrm{O}\right)\end{array}$ & $\begin{array}{c}\text { Arcilla } \\
(\%)\end{array}$ & $\begin{array}{c}\text { MOS } \\
(\%)\end{array}$ & $\begin{array}{c}\mathbf{P} \\
(\mu g \\
\left.g^{-1}\right)\end{array}$ & $\begin{array}{c}\mathbf{K} \\
(\mu g \\
\left.g^{-1}\right)\end{array}$ & $\begin{array}{c}\text { CIC } \\
\left(\mathrm{cmol}^{-1}\right. \\
\left.\mathrm{kg}^{-1}\right)\end{array}$ & $\begin{array}{l}\mathrm{Ca}^{2+} \\
(\mathrm{cmol} \\
\left.\mathrm{kg}^{-1}\right)\end{array}$ & $\begin{array}{l}\mathbf{M g}^{2+} \\
\left(\mathrm{cmol}^{-1}\right. \\
\left.\mathrm{kg}^{-1}\right)\end{array}$ & $\begin{array}{c}\mathbf{K}^{+} \\
(\mathrm{cmol} \\
\left.\mathrm{kg}^{-1}\right)\end{array}$ & $\begin{array}{c}\left(\mathrm{Al}^{3+}+\mathbf{H}^{+}\right) \\
(\mathrm{cmol} \mathrm{kg} \\
1)\end{array}$ \\
\hline $\mathbf{F e}$ & g & -0.13 & 0.40 & 0.14 & -0.03 & 0.21 & 0.02 & -0.03 & 0.35 & -0.09 & -0.01 \\
\hline Cu & $\mu g^{-1}$ & 0.11 & 0.19 & 0.08 & -0.03 & 0.06 & 0.07 & 0.04 & 0.40 & -0.05 & -0.20 \\
\hline Zn & $\mu g^{-1}$ & 0.10 & 0.38 & 0.25 & 0.08 & 0.16 & 0.19 & 0.18 & 0.36 & 0.03 & -0.17 \\
\hline Mn & $\mu g{ }^{-1}$ & -0.26 & & 0.14 & -0.10 & 0.14 & -0.13 & -0.12 & 0.04 & -0.19 & 0.00 \\
\hline Cd & $\mu g{ }^{-1}$ & 0.33 & 0.05 & 0.33 & 0.32 & 0.15 & 0.40 & 0.35 & 0.51 & 0.36 & -0.22 \\
\hline $\mathrm{Ni}$ & $\mu g^{-1}$ & -0.02 & 0.17 & 0.07 & -0.05 & 0.05 & -0.01 & -0.04 & 0.32 & -0.07 & -0.14 \\
\hline $\mathbf{P b}$ & $\mu g^{-1}$ & -0.08 & 0.44 & 0.18 & -0.11 & 0.20 & -0.03 & -0.03 & 0.07 & -0.17 & 0.07 \\
\hline
\end{tabular}

* Valores en negrita tienen correlación significativa entre variables $(\mathrm{p}<0.05)$
(1992). Las directivas de Kelly de la UE indica que los valores típicos en suelos no contaminados de cadmio están entre 0 y $1 \mu \mathrm{g} \mathrm{g}^{-1}$.

Los valores de níquel fluctuaron entre $1.64 \pm 2.13$ $\mu \mathrm{g} \mathrm{g}^{-1}$ y $43.03 \pm 2.13 \mu \mathrm{g} \mathrm{g}^{-1}$. Los valores obtenidos están por debajo del máximo permisible para Ni (30 a $100 \mathrm{\mu g} \mathrm{g}^{-1}$ ) (Cameron, 1992). Según las directivas de Kelly (Acevedo et al., 2005) los valores típicos de $\mathrm{Ni}$ en suelos no contaminados fluctúa entre 0 y $20 \mathrm{\mu g} \mathrm{g}^{-1}$. En esta categoría están Tumbes (Zarumilla), Piura (Morropón), Cajamarca, Amazonas (Bagua), San Martín, Huánuco (Leoncio Prado) y Junín. Suelos ligeramente contaminados (20 a $50 \mu \mathrm{g} \mathrm{g}^{-1}$ ) se encuentran en Tumbes (Tumbes), Piura (Huancabamba y Piura), Amazonas (Condorcanqui), Huánuco (Huamalíes) y Cuzco. No se hallaron suelos contaminados con níquel $\left(50-200 \mu \mathrm{g} \mathrm{g}^{-1}\right)$. No se encontraron referencias comparativas para estos elementos en suelos cacaoteros del Perú.

Por otro lado los valores de plomo estuvieron entre $5.52 \pm 0.41$ y $21.81 \pm 0.08 \mu g ~ g ~^{-1}$ correspondiente a San Martín (El Dorado) y Cuzco respectivamente. El promedio mayor de plomo $\left(21.81 \mathrm{\mu g} \mathrm{g}^{-1}\right)$ se encontró en la Zona Sur seguida de la Zona Norte $(11.20 \mu \mathrm{g} \mathrm{g}$ ${ }^{1}$ ) y Zona Centro $\left(8.66 \mu \mathrm{g} \mathrm{g}^{-1}\right)$. El Pb es ampliamente relacionado con la fracción disponible del suelo y los valores encontrados están por debajo del nivel excesivamente fitotóxico (50 y $100 \mu \mathrm{g} \mathrm{g}^{-1}$ ) de $\mathrm{Pb}$ en el suelo sugerido por Cameron (1992). Las directivas de Kelly (Acevedo et al., 2005) indican que los valores típicos de $\mathrm{Pb}$ en un suelo no contaminado fluctúan entre 0 y 200 $\mu \mathrm{g} \mathrm{g}^{-1}$. Los valores obtenidos en los suelos estudiados están dentro de esta categoría.

En el presente estudio los niveles de Cd y Pb están muy por debajo de estos rangos por lo que se puede inferir que los suelos muestreados no están 
contaminados por estos metales.

Los metales pesados y la profundidad del suelo

Los valores de metales pesados por profundidad de muestreo, se presentan en la Tabla 3. De forma general, no hubo diferencias estadísticas significativas ( $\mathrm{p}>0.05$ ) por profundidad de muestreo, con excepción del Cd. En la Figura 1 se presentan las tendencias de la variación de los valores de metales pesados en función a la profundidad. Los valores de Fe, Cu y Ni tienen la tendencia de ser mayores cuando se incrementa la profundidad, por el contrario el Zn, Mn, $\mathrm{Cd} \mathrm{y} \mathrm{el} \mathrm{Pb}$ presentan valores mayores en las capas superficiales. En general la concentración de Cd total decrece con la profundidad (Caridad-Cancela et al., 2005; He et al., 2015). Cameron (1992), indica un rango entre 3 a $10 \mu \mathrm{g} \mathrm{g}^{-1}$ de Cd y 50 y $100 \mu \mathrm{g} \mathrm{g}^{-1}$ de $\mathrm{Pb}$ para suelo superficial, como excesivamente fitotóxico.

Correlaciones de metales pesados y las principales características de los suelos estudiados

Los valores de la correlación de Pearson de los metales pesados estudiados con los principales atributos del suelo a profundidad de 0 a $20 \mathrm{~cm}$, se muestran en la Tabla 4. El Cd mostro correlación positiva y significativa $(\mathrm{p}<0.05)$ con el $\mathrm{pH}(\mathrm{r}=0.33)$, mientras que el Mn mostró una correlación negativa significativa $(\mathrm{r}=-0.26)$. El Cd mostró correlación positiva y significativa $(\mathrm{p}<0.05)$ con el $\mathrm{pH}, \mathrm{MOS}, \mathrm{P}$, CIC, $\mathrm{Ca}^{2+}, \mathrm{Mg}^{2+}$ y K $\mathrm{K}^{+}(\mathrm{r}=0.33,0.33,0.32,0.40,0.35$, 0.51 y 0.36 respectivamente) y con $\mathrm{Al}^{3+}+\mathrm{H}^{+}$la correlación fue negativa $(\mathrm{r}=-0.22)$. La movilidad y disponibilidad del $\mathrm{Cd}$ en el suelo esta principalmente controlada por el pH (Christensen, 1984; Alloway, 2013). Sin embargo, la presencia de materia orgánica y arcilla pueden tener efectos significativos en su disponibilidad (Degryse et al., 2009), explicando las correlaciones significativas con la CIC. Para el caso de los cationes su relación esta explicada debido a que la entrada de Cd en las raíces de las plantas es realizada en los mismos sitios donde $\mathrm{Ca}^{2+}, \mathrm{Mg}^{2+}$ y $\mathrm{K}^{+}$es absorbido (Kabata-Pendias, 2010). El Fe, Zn, Mn y $\mathrm{Pb}$ tuvieron correlación positiva $\mathrm{y}$ significativa $(\mathrm{p}<0.05)$ con el contenido de arcilla del suelo $(\mathrm{r}=0.40$, $0.38,0.51$ y 0.44 respectivamente). Se halló una correlación positiva y significativa del $\mathrm{Fe}, \mathrm{Cu}, \mathrm{Zn}, \mathrm{Cd}$ y Ni con el $\mathrm{Mg}^{2+}(r=0.35,0.40,0.36,0.51$ y 0.32 respectivamente). Sauve et al. (2000), indica que los metales pesados están en función al pH, el contenido de arcilla, materia orgánica, capacidad de intercambio catiónico y otros atributos del suelo. Así, Alloway (2013) indica que los factores más importantes en la adsorción de metales pesados son el contenido de arcilla, humus (materia orgánica) y óxidos. Este estudio muestra que estas relaciones positivas se observaron principalmente para el Cd y con los demás metales, determinados atributos fueron correlacionados positivamente.

\section{Conclusiones.}

Los suelos en el estudio presentan adecuadas condiciones físicas $\mathrm{y}$ químicas para el cultivo de cacao. Los valores de hierro, cobre, zinc, manganeso, cadmio, níquel y plomo, encontrados en los suelos muestreados estuvieron por debajo de los niveles considerados como fitotóxicos. Los valores promedio de hierro, zinc, manganeso, níquel y plomo fueron mayores en la zona sur, mientras que en la zona norte los valores de cobre y cadmio fueron mayores. A excepción del Cd, en los demás metales estudiados no encontramos diferencia estadística significativa $(\mathrm{p}>0.05)$ por profundidad de muestreo. Se observaron correlaciones positivas entre el $\mathrm{pH}$ y la
Figura 1. Mapa del estudio. Las zonas sombreadas indican la ubicación de las regiones y provincias consideradas en el presente estudio. 
presencia de cobre, zinc y cadmio en el suelo a excepción del plomo que tuvo una correlación negativa. El cadmio se correlacionó positivamente con el $\mathrm{pH}, \mathrm{MOS}, \mathrm{P}, \mathrm{K}$ disponible e intercambiable, CIC, $\mathrm{Ca}^{2+}, \mathrm{Mg}^{2+}$ y $\mathrm{K}^{+}$y negativamente con la acidez. A excepción del plomo, los demás metales se correlacionaron positivamente con la CIC y negativamente con la acidez intercambiable.

\section{Agradecimientos.}

Queremos agradecer al Programa Nacional de Innovación para la Competitividad y Productividad (Innóvate Perú), por el soporte financiero, Contrato $\mathrm{N}^{\mathrm{o}}$ 167-FINCyT-IB-2013. Al Departamento de Agricultura de los Estados Unidos-Servicio de Investigación Agrícola (USDA-ARS) por el soporte técnico y financiero. A Indian River Research and Education Center (IRREC), Universidad de Florida, USA. A la Embajada Norteamericana (INL) en LimaPerú por su continuo apoyo a la investigación y extensión en el Perú. Al Instituto de Cultivos Tropicales - ICT, por las facilidades de infraestructura y laboratorios. A todas las organizaciones cacaoteras del Perú que facilitaron el muestreo realizado.

\section{Literatura citada.}

Acevedo E., Carrasco M., León O., Silva P., Castillo G., Ahumada I., Borie G. \& González S. 2005. Informe de criterios de calidad de suelo agrícola. Servicio Agrícola y Ganadero, Chile. 205 p.

Aikpokpodion P. 2010. Nutrients Dynamics in Cocoa Soils, leaf and beans in Onto State, Nigeria. J. Agri. Sci. 1(1):1 -9 .

Aikpokpodion P. 2012. Assessment of Heavy Metals Movility in Selected Contaminated Cocoa Soils in Ondo State, Nigeria. Global Journal of Environmental Research 6: 30-35.

Alloway B.J. 2013. Heavy metals in Soils: Trace Metals and Metallloids in Soils and their Bioavailability, 3 ed. Springer.

Anderson J.M. \& Ingram J.S.I. 1993 Tropical soil biology and fertility: a handbook of methods. C.A.B. International. $221 \mathrm{p}$.

Augstburger F., Berger J., Censkowsky U., Heid, P., Milz, J. \& Streit C. 2000. Agricultura Orgánica en el trópico y subtrópico. In: Guía de 18 cultivos. 1ra Edición. Alemania. 24 p.

Bot A. \& Benites J. 2005. The Importance of Soil Organic Matter: Key to Drought-resistant Soil and sustained food production. 71-78 p.

Cameron R.E. 1992. Guide to site and soil description for hazardous waste site characterization. Vol 1: metals.EPA/600/4-91/029. 288p

Christensen T.H. 1984. Cadmium soil sorption at low concentrations. I.Effect of time, cadmium load, $\mathrm{pH}$, and calcium. Water, Air, and Soil Pollution, 21(1-4), 105114

Crozier J. 2012. Heavy metals in Cocoa. International Workshop on Possible EU regulations on cadmium in cocoaand chocolate products. Nature and Food Quality in the Netherlands (LNV), the European Cocoa Association (ECA) \& CAOBISCO.
Caridad-Cancela R., Paz-Gonzalez A. \& Abreu C.A., 2005. Total and extractable nickel and cadmium contents in natural soils. Commun. Soil Sci. Plant Anal. 36, $241 \mathrm{e} 252$.

Di Rienzo J.A., Casanoves F., Balzarin M.G., Gonzalez L., Tablada M. \& Robledo C.W., 2014. InfoStat versión 2014. Grupo InfoStat, FCA, Universidad Nacional de Córdoba, Argentina. URL http://www.infostat.com.ar

Degryse F., Smolders E. \& Parker D.R. 2009. Partitioning of metals (Cd, Co, $\mathrm{Cu}, \mathrm{Ni}, \mathrm{Pb}, \mathrm{Zn})$ in soils: concepts, methodologies, prediction and applications - A review. European Journal of Soil Science, 60(4): 590-612.

Giron C., Tortolero J., Hermoso D. \& Gonzalez I. 2001. Effect of various vegetable residues on cocoa shell compost. Agronomía Tropical 51: 549-562

He S., He Z., Yang X., Stoffella P.J. \& Baligar V.C. 2015. Soil Biogeochemistry, Plant Physiology, and Phytoremediation of Cadmium-Contaminated Soils. In: Sparks, D.L. (Ed.), Advances in Agronomy, pp. 135225.

Humani-Yupanqui H.A., Huauya-Rojas M.A., MansillaMinaya L.G., Florida-Rofner N. \& Neira-Trujillo G.M. 2012. Presencia de Metales pesados en el cultivo de cacao (Theobroma cacao L.) organico. Acta Agronomica, 61(4):339-344.

Kabata-Pendias. 2010. Trace Elements in Soils and Plants. 4 ed. CRC PRESS. 548p.

Khan A., Khan S., Khan M.A., Qamar Z. \& Wagas M. 2015. The uptake and bioaccumulation of heavy metals by food plants on plant nutrients, and associated health risk: a review. Environmental Science Pollution, 22:1377213799.

Lindsay W. \& Norvell W. 1978. Development of a DTPA soil test for zinc, iron, manganese, and copper. Soil Science Society of America Journal 42:421-428.

OEEE-MINAG. 2013. Series históricas de producción agrícola-Compendio estadístico. En http://frenteweb.minag.gob.pe/sisca/. Visitado el 17-052015.

Ogunlade M.O. \& Aikpokpodion P.O. 2006. Available Phosphorus and some micro-nutrient contents of cocoa soils in three cocoa growing ecological zones of Nigeria. Proceedings of 15th International Cocoa Research. Conference, Costa Rica.

Ololade I.A., Ajayi I.R., Gbadamosi A.E., Mohammed O.Z. \& Sunday A.G. 2010. A Study on Effects of Soil Physico-Chemical Properties on Cocoa Production in Ondo State. Modern Applied Science. 4(5):35-43

Puga S., Sosa M., Lebgue T., Quintana C. \& Campos A. 2006. Heavy Metals Pollution In Soils Damaged By Mining Industry. Ecología Aplicada, 5: 149-155

Prieto J., González C., Román A., \& Prieto F. 2009. Contaminación y fitotoxicidad en plantas por metales pesados provenientes de suelos y agua. Trop. Subtrop. Agroecosyst. 10(1): 29-44.

Rascio N. \& Navari-Izzo F. 2011. Heavy metal hyper accumulating plants: how and why do they do it? And what makes them so interesting? Plant Sci. 180: 169181.

Sauve S., Norvell W.A., Mcbride M. \& Hendershot W. 2000. Speciation and complexation of cadmium inextracted soil solutions. Environmental Science \& Technology, 34: 291-296. 
United States Environmental Protection Agency (USEPA). 1996. Method 3050 B: Acid digestion of sediment, sludges, and soils. CD-ROM. 3050B - 1. Revision 2, Washington, D.C.

United States Environmental Protection Agency (USEPA). 2002. Supplemental guidance for developing soil screening levels for superfund sites Available from http://www.epa.gov/superfund/health/conmedia/soil/ind ex.htm.
Violante A. \& Pigna M. 2002. Competitive sorption of arsenate and phosphate on different clay minerals and soils. Soil Science Society of American Journal, 66: 1788-1796.

Walkley A. \& Black I.A. 1934. An examination of the Degtjareff method for determining soil organic matter and a proposed modification of the chromic acid titration method. Soil Sci. 37: 29-38.

Yuan T.L. 1959. Determination of exchangeable hydrogen in soil by a titration method. Soil Science 88: 164-167. 
Tabla 1. Características físico-químicas de los suelos de plantaciones de cacao por Departamento y Provincias estudiadas de 0 a $20 \mathrm{~cm}$ de profundidad.

\begin{tabular}{|c|c|c|c|c|c|c|c|c|c|c|c|}
\hline$\frac{\text { Departamento }}{\text { Provincia }}$ & Textura & pH $\left(1: 2 \mathrm{H}_{2} \mathrm{O}\right)$ & Arcilla (\%) & MOS (\%) & $P\left(\mu g g^{-1}\right)$ & $K\left(\mu g g^{-1}\right)$ & $\begin{array}{c}\text { CIC } \\
\left(\mathrm{cmol} \mathrm{kg}^{-1}\right)\end{array}$ & $\begin{array}{c}\mathrm{Ca}^{2+} \\
\left(\mathrm{cmol} \mathrm{kg}^{-1}\right)\end{array}$ & $\begin{array}{c}\mathrm{Mg}^{2+} \\
\left(\mathrm{cmol} \mathrm{kg}^{-1}\right)\end{array}$ & $\begin{array}{c}\mathrm{K}^{+} \\
\left(\mathrm{cmol} \mathrm{kg}^{-1}\right)\end{array}$ & $\begin{array}{c}\left(\mathrm{Al}^{3+}+\mathrm{H}^{+}\right) \\
\left(\mathrm{cmol} \mathrm{kg}^{-1}\right)\end{array}$ \\
\hline \multicolumn{12}{|l|}{ Tumbes } \\
\hline umbes & Arcilloso & $7.62 \pm 0.01 \mathrm{a}$ & $9.28 \pm 0.71 \mathrm{a}$ & $2.04 \pm 0.11 \mathrm{a}$ & $.94 \pm 0.71 b$ & $121.88 \pm 4.75 a$ & $31.36 \pm 0.56 a$ & $23.04 \pm 0.67 a$ & $6.71 \pm 0.08 \mathrm{a}$ & $0.53 \pm 0.01 \mathrm{a}$ & $0.00 \pm 0.00 \mathrm{a}$ \\
\hline Zarumilla & Franco arcilloso & 01a & $.71 \mathrm{a}$ & $1.66 \pm 0.11 \mathrm{a}$ & $3.41 \pm 0.71 \mathrm{a}$ & $3245+475$ & $12.60 \pm 0.56 \mathrm{~b}$ & $7.78 \pm 0.67 b$ & $3.57 \pm 0.08 \mathrm{a}$ & $.31 \pm 0.01 a$ & $.00 \pm 0.00 a$ \\
\hline$\frac{\text { Piu }}{\text { Hua }}$ & rcillo Li & ( & & $3.03+0$ & 95 & $90.06+3.17 a$ & $7 \mathrm{a}$ & $15.68 \pm 0.45 a$ & .55 & la & \\
\hline Torropón & Arcilloso & 7 & & 2.92 & 27.7 & 0.0 & $7 a$ & $45 a$ & $5 a$ & $01 \mathrm{a}$ & $0.00 \mathrm{a}$ \\
\hline $\begin{array}{l}\text { Piura } \\
\text { Cajamarca }\end{array}$ & arcilloso & 7.02 & $0 \mathrm{a}$ & 3.65 & 30.47 & 121.88 & $4.11 \pm 0.16 a$ & $16.24 \pm 0.19 a$ & $5.66 \pm 0.02 a$ & $0.45 \pm 0.00 \mathrm{a}$ & $0.00 \pm 0.00 \mathrm{a}$ \\
\hline Jaen & $\begin{array}{l}\text { Franc } \\
\text { Limos }\end{array}$ & $8.29 \pm 0.01 \mathrm{a}$ & $39.44 \pm 0.48 a$ & $2.10 \pm 0.07 a$ & $7.40 \pm 0.46 b$ & $86.86 \pm 3.17 a$ & $32.60 \pm 0.37 a$ & $27.56 \pm 0.45 a$ & $2.82 \pm 0.05 b$ & $0.30 \pm 0.01 \mathrm{a}$ & $0.00 \pm 0.00 \mathrm{a}$ \\
\hline $\begin{array}{l}\text { San Ignacio } \\
\text { Amazonas }\end{array}$ & Arcillo Limoso & $8.07 \pm 0.01 \mathrm{a}$ & $37.95 \pm 0.71 a$ & $4.41 \pm 0.11 \mathrm{a}$ & $15.13 \pm 0.71 b$ & $161.54 \pm 4.75 a$ & $28.84 \pm 0.56 a$ & $25.60 \pm 0.67 a$ & $1.90 \pm 0.08 b$ & $0.53 \pm 0.01 \mathrm{a}$ & $0.00 \pm 0.00 a$ \\
\hline 3agu & oso & $40+$ & 10a & 3 & 5.7 & 1 & $.61 \pm$ & $16.08 \pm$ & $1.69 \pm 0.01 \mathrm{~b}$ & $.34 \pm$ & $0.18 \pm 0.04 a$ \\
\hline $\begin{array}{l}\text { Condorcanqui } \\
\text { San Martín }\end{array}$ & Arci & 6.0 & & 3.5 & & & $18 \mathrm{~b}$ & 8.3 & $1.23 \pm$ & 00a & $.10 \mathrm{a}$ \\
\hline Bellavista & Limoso & $7.67 \pm$ & $5.02 \pm 1.42 \mathrm{a}$ & 3.35 & 9. & $143.28+9.49 a$ & $41.09 \pm 1.12 \mathrm{a}$ & $38.69 \pm$ & $1.66 \pm$ & $0.62 \pm 0.03 a$ & $0.00 \pm 0.00 \mathrm{a}$ \\
\hline El D & Arc. Limoso & & & & & & 4 & 4. & $6 \mathrm{~b}$ & 3a & $.00 \mathrm{a}$ \\
\hline Iuallaga & Arcilloso & $7.78=$ & $42 \mathrm{a}$ & 2.25 & 6.76 & 99.6 & $32.15 \pm 1.12 \mathrm{a}$ & $30.14 \pm 1.35 a$ & $1.51 \pm 0.16 b$ & $0.40 \pm 0.03 a$ & $0.00 \pm 0.00 a$ \\
\hline $\begin{array}{l}\text { Mariscal } \\
\text { Cáceres }\end{array}$ & L & 7 & $28 \mathrm{a}$ & 2 & 1 & 0a & $.23 a$ & $.27 \mathrm{a}$ & $.03 b$ & $.01 \mathrm{a}$ & $.00 \mathrm{a}$ \\
\hline $\begin{array}{l}\text { Tocache } \\
\text { Huánuco }\end{array}$ & Franco & $5.57 \pm 0.00 \mathrm{~b}$ & $22.28 \pm 0.23 a$ & $1.88 \pm 0.04 a$ & $6.15 \pm 0.23 b$ & $63.36 \pm 1.59 a$ & $6.10 \pm 0.18 b$ & $2.99 \pm 0.23 b$ & $0.71 \pm 0.03 b$ & $0.23 \pm 0.00 \mathrm{a}$ & $0.25 \pm 0.10 \mathrm{a}$ \\
\hline Hua & $\begin{array}{l}\text { Franc } \\
\text { Limos }\end{array}$ & $\mathrm{Bb}$ & 3 & 2 & yb & 尚 & $2 \mathrm{~b}$ & 3. & $6 \mathrm{~b}$ & 3a & $0.62 \mathrm{a}$ \\
\hline $\begin{array}{l}\text { Leoncio Prado } \\
\text { Junin }\end{array}$ & Franco arcilloso & 5.76 & 27.46 & $2.07 \pm 0.07 a$ & 5.95 & $70.56 \pm 3.17 a$ & $10.96 \pm 0.37 b$ & $8.12 \pm 0.45 b$ & $1.21 \pm 0.05 b$ & $0.25 \pm 0.01 \mathrm{a}$ & $0.31 \pm 0.21 \mathrm{a}$ \\
\hline $\begin{array}{l}\text { Satipo } \\
\text { Cuzco } \\
\end{array}$ & Franco arcilloso & $6.86 \pm 0.01 \mathrm{a}$ & $28.52 \pm 0.35 a$ & $2.72 \pm 0.05 a$ & $11.42 \pm 0.35 b$ & $100.60 \pm 2.37 a$ & $15.76 \pm 0.28 b$ & $13.69 \pm 0.34 a$ & $1.77 \pm 0.04 \mathrm{~b}$ & $0.26 \pm 0.01 \mathrm{a}$ & $0.00 \pm 0.00 a$ \\
\hline La Convención & Franco arcilloso & $5.71 \pm 0.00 \mathrm{~b}$ & $34.46 \pm 0.28 a$ & $7.08 \pm 0.04 a$ & $29.16 \pm 0.28 \mathrm{a}$ & $64.80 \pm 1.90 \mathrm{a}$ & $13.03 \pm 0.23 b$ & $9.36 \pm 0.27 \mathrm{~b}$ & $1.88 \pm 0.03 b$ & $0.17 \pm 0.01 \mathrm{a}$ & $0.18 \pm 0.12 \mathrm{a}$ \\
\hline$P v$ & & 0.0002 & 0.4513 & 0.0288 & $<0.0001$ & 0.8512 & 0.0005 & 0.0012 & $<0.0001$ & 0.3198 & 0.9275 \\
\hline
\end{tabular}

*, Promedios unidos con igual letra en columna no difieren significativamente, según Scott \& Knott Alfa=0.05

\footnotetext{
${ }^{1}$ Instituto de Cultivos Tropicales, (ICT), Tarapoto, Perú. e.arevalo.ict@terra.com.pe.

${ }^{2}$ U.S. Department of Agriculture/Agricultural Research Service, Beltsville Agricultural Research Center, Beltsville, MD 20705, U.S.A.

${ }^{3}$ Indian River Research and Education Center (IRREC), University of Florida, Fort Pierce, Fl 34945, U.S.A.
} 